\begin{tabular}{|c|c|}
\hline A & $\begin{array}{c}\text { International Journal of Current Research } \\
\text { and Academic Review }\end{array}$ \\
\hline $\begin{array}{l}\text { EXCELLENT } \\
\text { PUBLISHERS } \\
\end{array}$ & $\begin{array}{c}\text { ISSN: 2347-3215 (Online) Volume } 7 \text { Number } 4 \text { (April-2019) } \\
\text { Journal homepage: http://www.ijcrar.com }\end{array}$ \\
\hline
\end{tabular}

doi: https://doi.org/10.20546/ijcrar.2019.704.011

\title{
Central Venous Catheter Tip Culture and Sensitivity Pattern from a Tertiary Care Hospital in Jammu Region, India
}

\author{
P. Sharma ${ }^{1 *}$, Perika ${ }^{1}$, S. Sudan ${ }^{1}$, N. Rajput ${ }^{1}$, S. Gupta ${ }^{1}$, Y. Bhandari ${ }^{1}$ and R. Tomar ${ }^{2}$ \\ ${ }^{1}$ Department of Microbiology, GMC, Jammu, India \\ ${ }^{2}$ Department of Microbiology, Santosh Medical College, Ghaziabad (U.P.), India \\ *Corresponding author
}

\begin{abstract}
The use of Central Venous Catheters (CVC) in critically ill patients often leads to Catheter Related Blood Stream Infections (CRBSI). Blood stream infections which are associated with catheter is associated with high morbidity and mortality. Aim of the study is to find the type of organisms isolated from Central venous catheter tip and to determine their antimicrobial susceptibility pattern. A total of 100 samples were received in our laboratory over a period of one year. Samples were processed; organisms were isolated and subjected to antimicrobial sensitivity according to standard laboratory procedures. AST interpretation was done according to latest CLSI Guidelines. Of the 100 samples received in our laboratory, 11(11\%) showed no growth or insignificant growth. $64 \%$ of the total samples were Gram Negative Bacilli and $23 \%$ were Gram Positive Cocci, 2\% were Candida albicans. Escherichia coli and Staphylococcus aureus were the most common organisms isolated among gram negative bacilli and gram positive cocci respectively. Sensitive drugs in case of gram positive cocci were vancomycin and linezolid and in case of gram negative bacilli were Colistin and polymyxin B.
\end{abstract}

\section{Article Info}

Accepted: 04 March 2019

Available Online: 20 April 2019

\section{Keywords}

Disinfectants, Variables, Process, Reactor, Automation, Design, Validation.

\section{Introduction}

Central venous catheter is used in care of critically ill patients to receive fluids and medicines. ${ }^{(1)}$ These intravascular devices act as vehicle for entry of microorganisms that colonize the skin adjacent to the site of entry or they may serve as foreign bodies, leading to catheter related blood stream infections (CRBSI). ${ }^{(2)}$

CRBSI is an important cause of healthcare associated infection to the blood stream. Several studies have shown increase in risk of bacteremia and sepsis following central venous catheter (CVC) insertion, therefore, prevention of CRBSI is an essential objective when these devices are used. Risk of infection depends on the material of catheter used, reason for its use, duration of catheterization ${ }^{(3)}$ and specific characteristics of the patient in whom catheter is placed. The administration of parenteral nutrition through intravascular catheters increases CRBSI risk. Local risk factors, such as poor personal hygiene, occlusive transparent dressing, and moisture around the exit site, Staphylococcus aureus nasal colonization, and contiguous infections support the role of bacterial colonization in the pathogenesis of CRBSI. Other risk factors for dialysis CRBSI include contamination of 
dialysate or equipment, inadequate water treatment, dialyzer re-use, older age, higher total intravenous iron dose, increased recombinant human erythropoietin dose, recent hospitalization or surgery. Hence, CRBSI rates can differ according to the institution and health-care institute understudy ${ }^{(4)}$.

CRBSI may be caused by Gram positive or Gram negative organisms but gram negative bacilli are responsible for a higher number of catheter related infections in Intensive Care Unit(ICU) than in Non- ICU patients ${ }^{(5)}$. Also, the relative risk for CRBSI is up to 64 times greater with CVCs than with peripheral venous catheters. Meta analytical study done at The Johns Hopkins University showed that bloodstream infections (BSI's) were the third leading cause of HospitalAcquired Infections (HAI's), with an attributable mortality rate of $12-25 \%{ }^{(6)}$. The diagnosis of CRBSI requires a positive culture of blood from a peripheral vein and clear evidence that the catheter is the source. CRBSI means a patient with an intravascular catheter has at least one positive blood culture obtained from a peripheral vein, clinical manifestations of infections, and no apparent source for the BSI, except the catheter.

This study is done with the aim to find the incidence of culture positive CVC tip, type of organisms isolated and their antibiotic sensitivity pattern. This can help in selection of the appropriate antimicrobial agent for prophylaxis and empirical therapy of CRBSI.

\section{Materials and Methods}

This analytical, cross sectional study was conducted over a period of one year in the Department of Microbiology, Government Medical College \& Hospital, Jammu (J\&K), India which is a tertiary care hospital having referral of Jammu province. Institute Ethics Committee approval was obtained for the study. All the tips from Intensive care unit (ICU) received in Microbiology Laboratory were included in this study.

The indications for central venous catheterization were for intravenous access and/or for central venous pressure monitoring. The catheter insertion was performed under strict aseptic precautions. A short section (approximately $5 \mathrm{~cm}$ ) of the catheter (including the area directly beneath the skin) was aseptically cut off and sent to the laboratory in a sterile tube for culture. This section of the catheter was rolled back and forth according to Extra Luminal Maki's Roll Plate Method (Figure.1) on the surface of Blood agar and MacConkey Agar plate with sterile forceps or straight wire and simultaneously Direct Gram Staining was performed. After overnight incubation, the colonies were counted. A positive culture was defined as a count more than or equal to 15 CFU/plate ${ }^{(3)}$. Significant colonies were further processed. Organisms were identified according to Colony Morphology, Gram Staining and Biochemical Tests.

Antibiotic Sensitivity was performed using Kirby-Bauer Disc Diffusion Method on Muller Hinton agar (HiMedia Labs). Different panels for Gram-Positive and Gram Negative bacteria were used as per Latest CLSI Guidelines (2019). Statistical analysis was performed using Microsoft Excel.

Antibiotic discs were procured from HiMedia Laboratories. The discs used for gram positive isolates were: Penicillin G (10 Units), Cefoxitin (30 mcg), Gentamicin (10 mcg), Ciprofloxacin (5 $\mathrm{mcg})$, Vancomycin (30 mcg), Linezolid (30 mcg).

Antibiotic discs used for gram- negative isolates were: Ampicillin (10 mcg), Piperacillin-tazobactam (100/10 $\mathrm{mcg}$ ), Cefuroxime (30 mcg), Cefoxitin (30 mcg), Cefepime (30 mcg),

Amikacin (30 mcg), Imipenem (10 mcg), Gentamicin (10 $\mathrm{mcg})$, Tobramycin $(10 \mathrm{mcg})$, Ciprofloxacin $(5 \mathrm{mcg})$, Cotrimoxazole $(1.25 / 23.5 \mathrm{mcg})$, Chloramphenicol (30 $\mathrm{mcg}$ ), Tetracycline $(30 \mathrm{mcg})$, Norfloxacin $(10 \mathrm{mcg})$, Amoxicillin/Clavulanic acid (20/10 mcg), Aztreonam (30 mcg).

\section{Results and Discussions}

A total of 100 samples were received in our laboratory for culture and sensitivity over a period of one year. Of the 100 samples, 11(11\%) showed no growth or insignificant growth and 89 showed significant growth showing a culture positivity of $89 \%$ (Figure.2). 64\% of pathogens isolated were gram negative bacilli, $23 \%$ were gram positive cocci, $2 \%$ were Candida albicans. Escherichia coli was the most common organism isolated among gram negative bacilli followed by Klebsiella pneumoniae. Among gram positive cocci, Staphylococcus aureus was the most common organism isolated. Distribution of Gram Negative Bacilli is given in (Table.1) and of Gram Positive Cocci given in (Table.2). The Antimicrobial Susceptibility Pattern is shown in (Table.3). 
Table.1 Distribution of Gram negative microorganisms

\begin{tabular}{|ll|}
\hline Organism & Number of Isolates $(\mathrm{n}=100)$ \\
\hline Escherichia coli & $22(22 \%)$ \\
\hline Klebsiella pneumoniae & $15(15 \%)$ \\
\hline Acinetobacter sp. & $12(12 \%)$ \\
\hline Pseudomonas sp. & $9(9 \%)$ \\
\hline Citrobacter sp. & $3(3 \%)$ \\
\hline Proteus vulgaris & $2(2 \%)$ \\
\hline Proteus mirabilis & $1(1 \%)$ \\
\hline
\end{tabular}

Table.2 Distribution of Gram positive microorganisms

\begin{tabular}{|ll|}
\hline Organism & Number of Isolates(n=100) \\
\hline Staphylococcus aureus & $16(16 \%)$ \\
\hline Enterococcus sp. & $5(5 \%)$ \\
\hline Micrococci & $2(2 \%)$ \\
\hline
\end{tabular}

Table.3 Antibiotic Susceptibility profile of organisms

\begin{tabular}{|lll|}
\hline Antibiotic & $\begin{array}{l}\text { Number of Sensitive } \\
\text { Isolates }(\%)\end{array}$ & $\begin{array}{l}\text { Number of Resistant Isolates } \\
(\%)\end{array}$ \\
\hline Penicillin & $4(19)$ & $17(81)$ \\
\hline Cefoxitin & $5(24)$ & $16(76)$ \\
\hline Gentamycin & $60(71)$ & $25(29)$ \\
\hline Ciprofloxacin & $50(59)$ & $35(41)$ \\
\hline Vancomycin & $20(95)$ & $1(5)$ \\
\hline Linezolid & $20(95)$ & $1(5)$ \\
\hline Ampicillin & $30(47)$ & $34(53)$ \\
\hline Piperacillin-Tazobactam & $40(63)$ & $24(38)$ \\
\hline Cefuroxime & $36(56)$ & $28(44)$ \\
\hline Cefepime & $35(55)$ & $29(45)$ \\
\hline Amikacin & $52(81)$ & $12(19)$ \\
\hline Imipenem & $45(70)$ & $19(30)$ \\
\hline Tobramycin & $45(70)$ & $19(30)$ \\
\hline Cotrimoxazole & $55(65)$ & $30(35)$ \\
\hline Chloramphenicol & $63(74)$ & $22(26)$ \\
\hline Tetracycline & $47(73)$ & $17(27)$ \\
\hline Amoxyclav & $34(53)$ & $30(47)$ \\
\hline Aztreonam & $44(69)$ & $20(31)$ \\
\hline Colistin & $64(100)$ & 0 \\
\hline Polymyxin B & $64(100)$ & 0 \\
\hline & & \\
\hline
\end{tabular}


Figure.1 Extra luminal Maki’s roll plate

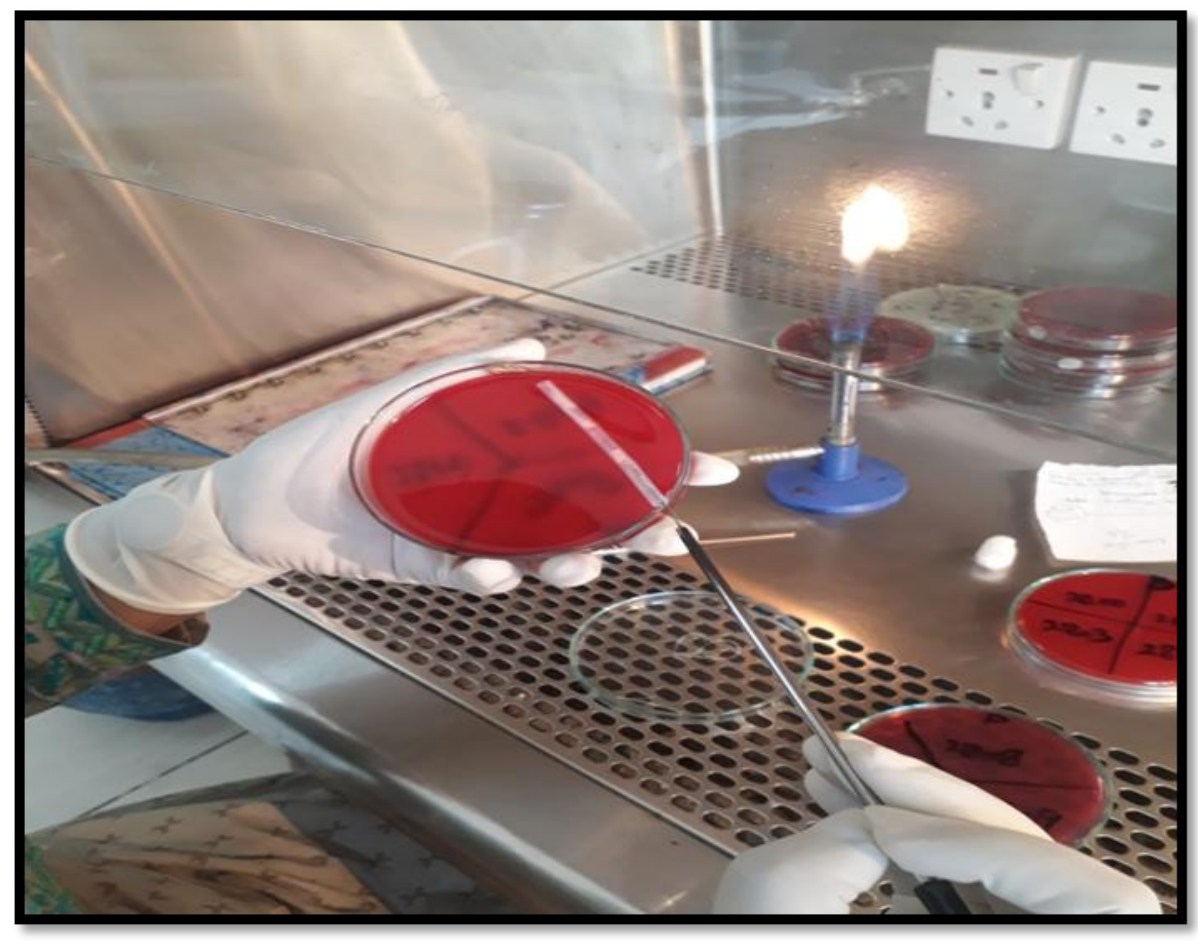

Figure.2 Culture positivity

Our study was done to investigate the rate of bacterial colonization and infection in central venous catheter tip culture together with antibiotic susceptibility profile of isolated bacteria. Central venous catheter is frequently used in hospital settings and colonization of CVC with a microorganism is one of the main risk factor for occurrence of CRBSI. In most of the studies done on central venous catheters, gram positive cocci were most

\section{$(\mathbf{n}=\mathbf{1 0}$}

frequently isolated ${ }^{(7-9)}$, but in our study most common organisms isolated were gram negative bacilli of which Escherichia coli was most predominant, consistent with some of the studies done recently ${ }^{(10,11)}$. In the study of Ramanathan Parameswaran et al., 2011 64\% of the pathogens causing CRBSI were Gram-positive, 36\% were Gram- negative. According to Krishnan et al., 2011 Gram positive cocci constituted $27 \%$ of isolates and 
gram negative bacilli were $56 \%$. The proportion of Gram negative CRBSI was much higher than that reported in western hospitals ${ }^{(12)}$. Siefert et al., 2003 showed coagulase- negative staphylococci was present in 50\% cases of CRBSI in their study ${ }^{(13)}$. According to Almuneef et al., 2006 total 50 CRBSI samples were received, $48 \%$ were polymicrobial, $32 \%$ were due to Gram - negative bacilli, and $10 \%$ were due to Gram positive organisms. In our study among infections caused by gram positive isolates Staphylococcus aureus was the most common organism. Various literatures shows that common organisms causing CVC associated infections are Staphylococcus aureus, Candida sp. and Gram negative bacilli ${ }^{(14,15)}$ and our study also correlated with these findings.

Our study shows most sensitive drugs among Gram negative isolates were Colistin, Polymyxin B, and intermediate sensitive were Imipenem, Amikacin, Aztreonam, Piperacillin -tazobactam and Tobramycin. Antibiotics like Penicillin, Ampicillin, and Cefepime showed high degree of resistance. Whereas study of Sapkota et al., 2017 concluded that chloramphenicol was the most sensitive drug, Piperacillin -tazobactam showed decrease sensitivity and antibiotics like Gentamicin and Amikacin as resistant drugs. Colistin also showed 100\% sensitive in their study, which correlated with our study. (16)

Among Gram positive cocci, most effective drugs were Vancomycin and Linezolid, which was in concordance with the study of Mansur et al., 2014; where vancomycin was the $100 \%$ sensitive drug. ${ }^{(17)}$

\section{Conclusion}

The present study has highlighted shift in colonizers of central venous catheter predominantly towards Gram negative bacilli and their appropriate antibiotic sensitivity profile. These findings can help in implementing educational and training programs on CRBSI for health personnel who can enable better management of these devices with regard to prevention, diagnosis and treatment. Hence, in order to reduce the mortality and morbidity of CRBSI, active intervention is required to ascertain the signs of sepsis in the patient at the earliest and send the properly collected samples at appropriate time for an early diagnosis.

\section{References}

1. O'Grady NP, Alexander M, Dellinger EP,
Gerberding JL, Heard SO, Maki DG, et al., Guidelines for the prevention of intravascular catheter-related infections. Infect Control Hosp Epidemiol 2002; 23: 759-69.

2. Wenzel RP, Edmond MB. The impact of hospital-acquired bloodstream infections. Emerg Infect Dis 2001; 7: 174-7.

3. Zingg W, Sax H, Inan C, Cartier V, Diby M, Clergue F, et al. Hospital-wide surveillance of catheter-related bloodstream infection: From the expected to the unexpected. J Hosp Infect 2009; 73:41-6.

4. Maki DG, Weise CE, Sarafin HW. A semiquantitative culture method for identifying intravenous-catheter- related infections. $N$ Engl $J$ Med 1977; 296:1305-9.

5. Eggiman P, Pittet D. Overview of catheter related infections with special emphasis on prevention based on educational programs. Clin Microbiol Infect 2002; 8:295-309.

6. Maki DG, Kluger DM, Crnich CJ. The risk of bloodstream infection in adults with different intravascular devices: a systematic review of 200 published prospective studies. Mayo Clin Proc.2006; 81:1159-71.

7. Winn WC, Koneman EW. Koneman's color atlas and textbook of diagnostic microbiology. Philadelphia: Lippincott Williams \& Wilkins; 2006.

8. Meadows C, Creagh-Brown B, Nia T, Bonnici K, Finney S. Definition of catheter-related bloodstream infection as a quality improvement measure in intensive care. CriticalCare. 2009; 13:191.

9. Tullu MS, Deshmukh CT, Baveja SM. Bacterial profile and antimicrobial susceptibility pattern in catheter related nosocomial infections. Postgrad Med J 1998; 44:7-13.

10. Pérez-Granda MJ, Guembe M, Cruces R, Barrio JM, Bouza E. Assessment of central venous catheter colonization using surveillance culture of withdrawn connectors and insertion site skin. Critical Care. 2016; 20:32.

11. Hodzic S, Tihic N, Smajic J, Omerbegovic M, Sljivic M. Frequency of the central venous catheter colonization in surgical intensive care unit. Medicinski arhiv. 2010; 64:245-7.

12. Krishnan RG, Dorairajan Sureshkumar. Changing Trends in Antimicrobial Susceptibility and Hospital Acquired Infections over an year period in a Tertiary care Hospital in Relation to introduction of an Infection Control Programme. 
JAPI. 2011

13. Seifert H, Cornely O, Seggewiss K, Decker M, Stefanik D, Wisplinghoff H.Bloodstream infection in neutropenic cancer patients related to short-termnontunnelled catheters determined by quantitative blood cultures, differential time to positivity, and molecular epidemiological typing with pulsed- field gel electrophoresis. $J$ Clin Microbiol 2003; 41:118-23.

14. Naveen G, G Latha and Nagraj C. Bacteriological Study of Central Line Associated Blood Stream Infection at a Tertiary Care Hospital. Int J Curr Microbiol App Sci 2016; 5:645-49.
15. Pascual A. Pathogenesis of catheter-related infections: lessons for new designs. Clin Microbiol Infect 2002; 8:256-64.

16. Mansur FJ, Barai L, Karim MM, Haq JA, Fatema K, Faruq MO. Intravascular catheter related infections and antimicrobial susceptibility pattern of isolated bacteria in a tertiary care hospital of Bangladesh. Indian $J$ Med Microb 2014; 32(1):68-71.

17. Sapkota J, Mishra B, Jha B, Sharma M. Bacteriological profile and their antimicrobial susceptibility pattern in central venous catheter tip culture. J Pathol Nepal 2017; 7:1059-61.

\section{How to cite this article:}

Sharma, P., Perika, S. Sudan, N. Rajput, S. Gupta, Y. Bhandari and Tomar, R. 2019. Central Venous Catheter Tip Culture and Sensitivity Pattern from a Tertiary Care Hospital in Jammu Region, India. Int.J.Curr.Res.Aca.Rev. 7(4), 101-106. doi: https://doi.org/10.20546/ijcrar.2019.704.011 